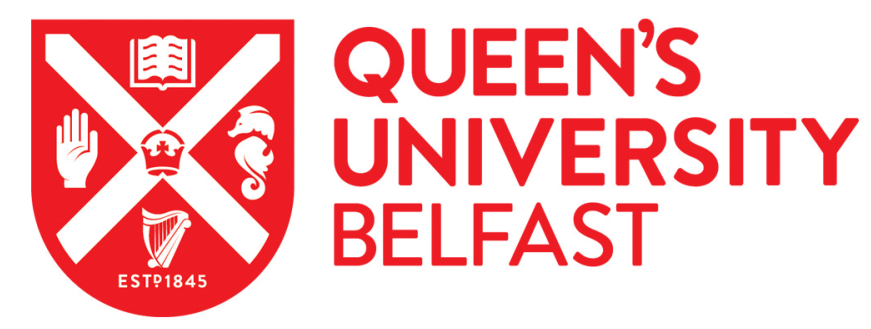

\title{
The diagnosis and management of respiratory tract fungal infection in cystic fibrosis: A UK survey of current practice
}

Boyle, M., Moore, J. E., Whitehouse, J. L., Bilton, D., \& Downey, D. G. (2018). The diagnosis and management of respiratory tract fungal infection in cystic fibrosis: A UK survey of current practice. Medical Mycology. https://doi.org/10.1093/mmy/myy014

Published in:

Medical Mycology

Document Version:

Peer reviewed version

Queen's University Belfast - Research Portal:

Link to publication record in Queen's University Belfast Research Portal

Publisher rights

(c) 2018 The Authors. This work is made available online in accordance with the publisher's policies. Please refer to any applicable terms of use of the publisher.

\section{General rights}

Copyright for the publications made accessible via the Queen's University Belfast Research Portal is retained by the author(s) and / or other copyright owners and it is a condition of accessing these publications that users recognise and abide by the legal requirements associated with these rights.

Take down policy

The Research Portal is Queen's institutional repository that provides access to Queen's research output. Every effort has been made to ensure that content in the Research Portal does not infringe any person's rights, or applicable UK laws. If you discover content in the Research Portal that you believe breaches copyright or violates any law, please contact openaccess@qub.ac.uk. 


\section{The Diagnosis and Management of Respiratory Tract Fungal Infection in Cystic Fibrosis - A UK Survey of Current Practice}

M Boyle ${ }^{1}$, JE Moore ${ }^{1}$, JL Whitehouse ${ }^{2}$, D Bilton ${ }^{3}$, DG Downey ${ }^{1,4}$

${ }^{1}$ Northern Ireland Regional Adult CF Centre, Belfast City Hospital, Belfast, N. Ireland, UK

${ }^{2}$ Heartlands Hospital, Birmingham, UK

${ }^{3}$ Royal Brompton Hospital, London, UK

${ }^{4}$ Centre for Experimental Medicine, Queen's University of Belfast, Belfast, N. Ireland, UK

Correspondence to:

Dr Damian Downey

Centre for Experimental Medicine, Queen's University of Belfast, Belfast, N. Ireland Email: d.downey@qub.ac.uk, Phone +442890329241

Short title: Diagnosis and management of respiratory fungal infection in cystic fibrosis

Key Words: Cystic fibrosis, aspergillus, itraconazole, treatment. 


\section{Abstract}

Aspergillus fumigatus is commonly found in the airways of patients with Cystic Fibrosis, (CF) and allergic bronchopulmonary aspergillosis (ABPA) is the most recognised associated clinical condition. However accurate diagnosis remains challenging and there is a paucity of clinical trials to guide clinical management of fungal disease. The aim of this survey was to assess the variability in current practice across the UK in diagnosis and management of fungal lung disease in CF patients. A 21 question anonymous online survey was sent to 94 paediatric and adult CF consultants in the UK.

The response rate was $60.6 \%$ ( 32 adult physicians, 25 paediatricians) with 55 full and 2 partially completed surveys. For a first diagnosis of ABPA 20 (35.1\%) treat with prednisolone alone, $38(66.7 \%)$ use prednisolone with itraconazole and 2 (3.5\%) choose voriconazole. Only $5(8.8 \%)$ treat with prednisolone alone for a $1^{\text {st }}$ relapse, 33 (58\%) used prednisolone with itraconazole.

To reduce treatment, 21 (36.8\%) decrease steroids to zero over time and maintain azole therapy, 18 (31.6\%) stop the azole and steroid after a fixed time and 5 (8.8\%) stop azole after a fixed time and maintain a small steroid dose. Thirty-eight (66.7\%) respondents believe Aspergillus colonisation of the airway can cause clinical deterioration and $37(66.1 \%)$ would treat this. Scedosporium apiospermum infection has been diagnosed and treated by 35 (61.4\%) of respondents.

Results of this survey highlight the variance in clinical practice and the limited evidence available to guide management of fungal infection in CF. 


\section{INTRODUCTION}

People with Cystic Fibrosis (PWCF) are extremely vulnerable to respiratory tract infections. Although infections are mainly bacterial in origin, with particular emphasis on $P$. aeruginosa, fungal species are also commonly cultured. Aspergillus fumigatus is the most common fungal species found in the sputum of PWCF, with an estimated prevalence of $6-57 \% .{ }^{1-4}$ Allergic bronchopulmonary aspergillosis (ABPA) is the main clinical manifestation. However, asymptomatic colonisation, Aspergillus bronchitis and Aspergillus sensitisation are also recognized as part of the disease spectrum. $2,5,6$

The most widely accepted criteria used to diagnose ABPA in PWCF were proposed at the 2003 CF Foundation Consensus Conference. ${ }^{7}$ However since 2005, 50\% of publications regarding ABPA have used alternative diagnostic criteria and the prevalence of ABPA differed when studies were stratified according to the criteria used for diagnosis. ${ }^{8}$ Diagnosis of Aspergillus sensitisation and bronchitis can also be challenging due to an overlap in symptoms and diagnostic markers. The prevalence of Aspergillus bronchitis in adult CF patients has been estimated at $30 \%$ and sensitisation to Aspergillus between $15-65 \% .{ }^{6,8}$

Despite the prevalence of fungal related infection in PWCF there is a paucity of randomised controlled trials (RCTs) to guide diagnosis and management. Although corticosteroids are the mainstay of treatment for ABPA, there is little evidence to guide dosing and duration of treatment, despite the significant associated side 
effects. ${ }^{9}$ A 2014 systematic review of ABPA treatment (in patients with and without CF) demonstrated limited evidence for anti-fungal therapy. However a trend towards improved symptoms, frequency of exacerbations and lung function was observed. ${ }^{10}$ The Infectious Diseases Society of America (IDSA) 2016 guidelines for treatment of ABPA and Aspergillus bronchitis make weak recommendations due to low quality evidence. ${ }^{11}$ There are no evidence based guidelines on the diagnosis and management of other less commonly isolated fungi such as Scedosporium apiospermum.

Our aim was to obtain data on how respiratory tract fungal infections are diagnosed, monitored and managed in UK CF centres.

\section{METHODS}

A 21 question anonymous electronic survey was emailed to all known 94 consultants affiliated with recognised UK CF centres. The survey explored the criteria used to diagnose and screen for ABPA, how ABPA is treated (and on first and second relapse), treatment duration/reduction and routine monitoring carried out whilst on treatment. We asked if CF physicians would treat Aspergillus colonization, Aspergillus bronchitis or Aspergillus sensitisation not meeting ABPA criteria. Finally, we requested details of experience in managing Scedosporium apiospermum and their views on RCTs in ABPA, non ABPA Aspergillus conditions and other fungal infections. We compared adult to paediatric responses using Chi square analysis. 


\section{RESULTS}

The response rate was $60.6 \%$ (55 fully and 2 partially completed). There were 32 (56.1\%) responses from adult physicians and 25 (43.9\%) from paediatricians. The number of PWCF attending these centres varied from 25-600, (median 205).

\section{Criteria used to diagnose ABPA}

Physicians were asked to choose criteria used to diagnose ABPA. Fifty-six (98.2\%) of respondents reported using total serum IgE; 53 (93\%) new chest X-ray abnormalities; 49 (86\%) acute deterioration not attributable to another cause; 41 (71.9\%) peripheral blood eosinophilia; 40 (70.2\%) Aspergillus serum precipitins; 35 (61.4\%) Aspergillus culture; 26 (45.6\%) Aspergillus specific IgE and 7 (12.3\%) skin prick testing. Thirty five $(61.4 \%)$ confirmed they had access to galactomannan testing in local laboratories, however only 8 (14\%) would use it for this indication.

All 57 (100\%) reported measuring total serum IgE, 54 (94.7\%) measured specific Aspergillus IgE and 43 (75.4\%) measured specific Aspergillus IgG when screening for ABPA. Almost all respondents, 56 (98.2\%), screen annually for ABPA. In the additional comments section, $4(7 \%)$ respondents stated they check an eosinophil count, $3(5 \%)$ send a routine sputum culture for Aspergillus and 2 (3.5\%) perform a routine CXR.

\section{$\underline{\text { Treatment }}$}

In the treatment of a first diagnosis of ABPA, 38 (66.7\%) physicians would use 
prednisolone alongside itraconazole (19 (33.7\%) using capsules and 19 (33.7\%) using itraconazole liquid). Twenty (35.1\%) respondents reported using prednisolone alone. Prednisolone with voriconazole was only chosen by 2 (3.5\%). Unfortunately to allow additional comments participants could check more than one answer. Additional reported treatments included, methylprednisolone, posaconazole, nebulized amphotericin and IV caspofungin.

There was a significant difference in adult and paediatric physician treatment choices. For a first diagnosis of ABPA, 15 (47\%) of adult physicians treat with prednisolone alone, compared with just 5 (20\%) paediatricians $(p<0.0001)$. Prednisolone with itraconazole capsules was chosen by $13(41 \%)$ adult physicians and 6 (24\%) paediatricians. Prednisolone with itraconazole liquid was more popular among paediatricians with $15(60 \%)$ choosing this as first line treatment, compared to $4(12.5 \%)$ adult physicians. Finally, no adult physicians reported using voriconazole but $2(8 \%)$ paediatricians would use prednisolone with voriconazole. (Figure 1)

For a first relapse of $A B P A, 33(58 \%)$ reported using prednisolone alongside itraconazole (19 (33.3\%) using liquid and 14 (24.6\%) using capsules). Prednisolone with voriconazole was chosen by $7(12.3 \%)$, prednisolone alone was chosen by 5 $(8.8 \%)$ respondents and $4(7 \%)$ physicians stated they would use prednisolone and posaconazole.

There was a significant difference in the adult and paediatric physician choices in the management of a first relapse. Four (12.5\%) adult physicians chose prednisolone 
alone, compared with only $1(4 \%)$ paediatrician $(p<0.0001)$. Prednisolone and itraconazole was used by 20 (62.5\%) of the adult physicians (11 choosing capsules and 9 liquid), compared to 13 (52\%) of the paediatricians (3 (12\%) choosing capsules and 10 (40\%) liquid). Prednisolone and voriconazole was used by 5 (16\%) adult physicians and $2(8 \%)$ paediatricians. (Figure 2)

For a second relapse of ABPA, 19 (33.3\%) used prednisolone with voriconazole. Prednisolone with itraconazole was chosen by 15 (26\%). Four (7\%) respondents stated they would choose prednisolone plus posaconazole, $4(7 \%)$ would treat the same as a first relapse, $3(5 \%)$ reported the use of methylprednisolone and $3(5 \%)$ would more likely use voriconazole. Prednisolone alone was reported as treatment of choice by one physician.

\section{Nebulised Amphotericin and Omalizumab}

Thirty three (57.9\%) respondents have had experience of using nebulised amphotericin whilst 32 (56.1\%) had experience of Omalizumab. Eleven (19.3\%) respondents had used neither.

\section{$\underline{\text { Treatment monitoring }}$}

All 57 (100\%) physicians would monitor total serum IgE, 47(82.5\%) specific Aspergillus IgE and 28 (49.1\%) specific Aspergillus IgG. Spirometry is monitored by 54 (94.7\%) and chest X-ray by 38 (66.7\%). Additionally, 5 physicians reported monitoring eosinophil count and 2 stated they would monitor a chest X-ray only if there were $\mathrm{X}$-ray changes at the time of diagnosis. 
The majority of physicians, 48 (84\%), monitor azole levels if used in treatment.

\section{Reducing Treatment when Clinically Stable on Steroid and Azole Therapy}

Twenty one (36.8\%) would reduce steroids to zero over time and maintain azole therapy, 18 (31.6\%) would stop the azole and steroid after a fixed time and 5 (8.8\%) would stop the azole after a fixed time (e.g. 3 or 6 months) and maintain a small steroid dose. Additional comments were permitted to obtain further detail, 10 would reduce steroid to zero then stop the azole later (3 would stop the azole at 3-6 months and 7 based the timescale of stopping the azole on each patient's response and stability). (Figure 3)

\section{Non ABPA Aspergillus conditions}

Thirty eight (66.7\%) respondents believed that chronic Aspergillus colonisation of the airways can cause clinical deterioration, whereas 15 (26.3\%) were unsure. Thirty seven (66.1\%) respondents reported they would treat this, $3(5.4 \%)$ would not and 16 (28.6\%) were unsure. Aspergillus bronchitis has been treated by 42 respondents (73.7\%) and 6 (10.5\%) have not. Thirty (52.6\%) physicians believe that Aspergillus sensitisation (not meeting ABPA criteria) of the airways can cause clinical deterioration, 3 (5.3\%) did not and 19 (33.3\%) were unsure. Twenty seven (49.1\%) stated they would treat it and $12(21.8 \%)$ would not.

\section{Scedosporium apiospermum}

Thirty five (61.4\%) had experience in treating patients with Scedosporium 
apiospermum, 22 (38.6\%) did not. Voriconazole was the treatment of choice by 21 (46.7\%) and 13 (28.9\%) would use prednisolone and voriconazole. Four physicians stated they would use posaconazole to treat Scedosporium apiospermum.

\section{Further Research}

Forty six (80.7\%) respondents believed that RCTs were required in ABPA, 43 (75.4\%) for non-ABPA Aspergillus conditions and 33 (57.9\%) for other fungi.

However challenges were noted, 'very difficult due to diverse diagnostic criteria', 'this would be difficult as ABPA is difficult to characterise at least in paediatrics' and, 'we have attributed serious and rapid clinical decline to a variety of fungal infections that fall outside guideline ABPA diagnostic criteria.'

\section{DISCUSSION}

This survey was designed to gain an overview of the diagnosis and clinical management of fungal disease in UK CF Centres and determine some of the challenges that arise with recurrent disease. To our knowledge this is the largest survey carried out in the UK to date. A smaller and less comprehensive survey in 2001 also reported variability in diagnostic criteria and treatment. ${ }^{12}$

Aspergillus fumigatus can cause a spectrum of hypersensitivity responses and disease phenotypes. A recent review described a wide range of criteria used in the 
diagnosis of ABPA. ${ }^{8}$ Our results show that total serum IgE was the most commonly used test (98\%) but there is individual variability in immune response which results in difficulty defining a value to be used in diagnostic criteria. ${ }^{13}$ Interestingly $67 \%$ have access to galactomannan testing in their local laboratory but only $13.3 \%$ use it to aid in diagnosis. This may be due to physician uncertainty in the interpretation of results and the implication for treatment. Serum galactomannan testing has a low sensitivity ${ }^{14,15}$ which may limit its use as a diagnostic tool. Also there are conflicting reports of the usefulness of sputum galactomannan testing as a diagnostic aid in Aspergillus related infection. ${ }^{3,16}$ However, sputum galactomannan with Aspergillus real time PCR and serological tests can be used in classifying Aspergillus disease phenotypes. $^{2}$

Skin prick testing for an immediate IgE mediated response is a sensitive marker of Aspergillus sensitisation and along with an elevated total serum IgE is useful in the diagnosis of ABPA. 9 Although part of the 2003 Consensus Conference recommendations only $13 \%$ of our responding physicians employ it. The reasons were not documented but this may have been due to test availability and a positive result is not specific for ABPA. ${ }^{17}$ The majority of respondents (72\%) use peripheral blood eosinophilia as a diagnostic aid. This is widely available and inexpensive but has limited value in ABPA diagnosis. ${ }^{7,18}$ Newer biomarkers have been evaluated, but are not yet established or widely used. IgE specific to certain recombinant Aspergillus allergens, Thymus activation-regulated chemokine (TARC/CCL17) and basophil activation testing, may become useful biomarkers in diagnosis and for distinguishing ABPA from non ABPA Aspergillus related conditions. ${ }^{13}$ 19, 20 
There was also considerable variation in ABPA treatment practices. For a first diagnosis, the most common regime was prednisolone and itraconazole, (66.7\%) followed by prednisolone alone (35.1\%). As the survey progressed from first diagnosis to recurrent ABPA relapse, the number of additional comments increased, highlighting an increasing variation in practice in more complex cases of ABPA. Additional differences in adult and paediatric treatment regimens have not been reported before. Adult physicians were more likely to use prednisolone alone to treat a first diagnosis of ABPA whereas paediatricians prefer the addition of itraconazole.

There have been RCTs of itraconazole in ABPA for patients with asthma. ${ }^{21}$ Antifungal treatment has considerable side effects, drug interactions and challenges with bioavailability. ${ }^{22}$ Drug monitoring is recommended ${ }^{11}$ but $23 \%$ of respondents do not monitor levels. Sub therapeutic azole levels may play a role in the development of azole resistance. Case reports described azole resistance in ABPA and Aspergillus bronchitis in 2009. ${ }^{23}$ In a study of 519 patients with Aspergillus fumigatus isolates there was a $5 \%$ frequency of itraconazole resistance with $65 \%$ cross-resistance to voriconazole. ${ }^{24}$ In a CF study a $4.6 \%$ rate of itraconazole resistance was noted and all of these isolates also showed reduced susceptibility to posaconazole. ${ }^{25}$ Higher rates of azole resistance were also found in those recently exposed to itraconazole. ${ }^{25}$ The CF Trust laboratory standards currently recommend susceptibility testing when there is repeated isolation of Aspergillus sp. despite long-term azole treatment. ${ }^{26}$ Pulsed methylprednisolone, caspofungin and nebulised amphotericin are utilised in more 
complex cases. This is based on clinical experience rather than significant published data.

There was variation in treatment practice when PWCF were clinically stable. The majority response was to reduce the steroid to zero and maintain the azole. This would be stopped at a later date, usually at 3-6 months but others stating 'gradually' and 'as per clinical response'. With the potential for relapse balanced with toxicity of prolonged treatment there is a pressing need for guidance in both treatment and duration.

Omalizumab is a monoclonal anti-IgE antibody that can improve control in allergic asthma. ${ }^{27}$ A RCT in PWCF was prematurely terminated due to challenges with recruitment and retention however a case report series described an improvement in $\mathrm{FEV}_{1}$ and symptoms with a reduction in steroid use in children. ${ }^{28}$ Over half of our respondents have previous experience of using it and it may be beneficial to collate this experience for regimen guidance in complex cases.

It is increasingly recognized that Aspergillus species can cause clinical deterioration in PWCF even when ABPA diagnostic criteria are not fulfilled. ${ }^{2}$ Thirty-seven percent of our respondents reported that they would treat a clinical deterioration from Aspergillus colonisation, 49\% would consider treating Aspergillus sensitisation and 74\% would treat Aspergillus bronchitis. Aspergillus sensitisation is defined as a positive skin prick test to Aspergillus antigens or an elevated serum Aspergillus IgE level. ${ }^{1,8}$ An observational study over two years described a decline in $\mathrm{FEV}_{1}$ and an 
increase in IV antibiotic days for patients sensitised to Aspergillus. ${ }^{29}$ Aspergillus bronchitis is a clinical deterioration associated with positive Aspergillus cultures but low or minimally elevated markers of sensitisation. ${ }^{2,5,30}$ Shoseyov et al described 6 patients with CF and positive sputum for Aspergillus fumigatus in keeping with this diagnosis. Following antifungal therapy there was a clinical improvement in symptoms, weight and $\mathrm{FEV}_{1}$ in all 6 patients. ${ }^{30}$ However, evidence remains limited and there is uncertainty regarding the immediate and long-term clinical effects of colonisation and sensitisation. ${ }^{6,30}$

Scedosporium apiospermum is a saprophytic filamentous fungus and is the second most frequent fungus to colonise PWCF. ${ }^{31,32}$ Case reports suggest that it may have a pathogenic role in CF. ${ }^{33,34}$ However Parize et al did not demonstrate a clinical deterioration with colonisation or sensitisation. ${ }^{35}$ There was uncertainty among survey respondents regarding the significance and treatment of Scedosporium, however the majority (61.4\%) have some experience in treating patients.

This survey has highlighted a variety of challenges in CF respiratory tract fungal disease. Designing and executing prospective RCTs in fungal disease will be challenging, therefore a rethink of trial design may be required. Perhaps exploring registry data, CF centres clustered for comparative treatment effectiveness or adaptive trial designs are some possibilities. However we must rise to the challenge of understanding our treatment choices and establishing an evidence base for them. 
ACKNOWLEDGEMENTS

\section{Conflict of interest}

All the authors have no conflicts of interest to report. 


\section{References:}

1. Burgel PR, Paugam A, Hubert D, Martin C. Aspergillus fumigatus in the cystic fibrosis lung: pros and cons of azole therapy. Infect Drug Resist. 2016; 9: 229238.

2. Baxter CG, Dunn G, Jones AM et al. Novel immunologic classification of aspergillosis in adult cystic fibrosis. J Allergy Clin Immunol. 2013; 132: 560566.

3. Bakare N, Rickerts V, Bargon J, Just-Nubling G. Prevalence of Aspergillus fumigatus and other fungal species in the sputum of adult patients with cystic fibrosis. Mycoses. 2003; 46: 19-23.

4. Paugam A, Baixench MT, Demazes-Dufeu N et al. Characteristics and consequences of airway colonization by filamentous fungi in 201 adult patients with cystic fibrosis in France. Med Mycol. 2010; 48; S32-S36.

5. Jones AM, Horsley A, Denning DW. What is the importance of classifying Aspergillus disease in cystic fibrosis patients? Expert Rev Respir Med. 2014; 8: 389-392.

6. Armstead J, Morris J, Denning DW. Multi-country estimate of different manifestations of aspergillosis in cystic fibrosis. PLoS One. 2014; 9: e98502.

7. Stevens DA, Moss RB, Kurup VP et al. Allergic Bronchopulmonary Aspergillosis in Cystic Fibrosis-State of the Art: Cystic Fibrosis Foundation Consensus Conference. Clin Infect Dis. 2003; 37: S225-264.

8. Maturu VN and Agarwal R. Prevalence of aspergillus sensitization and allergic 
bronchopulmonary aspergillosis in cystic fibrosis: systemic review and meta analysis. Clin Exp Allergy. 2015; 45: 1756-1778.

9. Patterson K and Streak M. Allergic Bronchopulmonary Aspergillosis. Proc Am Thorac Soc. 2010; 7: 237-244.

10. Moreira AS, Silva D, Reis Ferreira AR and Delgado L. Antifungal treatment in allergic bronchopulmonary aspergillosis with and without cystic fibrosis: a systematic review. Clin Exp Allergy. 2014; 44: 1210-1227.

11. Patterson TF, Thompson GR, Denning DW et al. Practice Guidelines for the Diagnosis and Management of Aspergillosis: 2016 Update by the Infectious Diseases Society of America. Clin Infect Dis. 2016; 63: e1-60.

12. Cunningham S, Madge SL, Dinwiddie R. Survey of criteria used to diagnose allergic bronchopulmonary aspergillosis in cystic fibrosis. Arch Dis Child. 2001; $84: 89-93$.

13. Delhaes L, Frealle E, Pinel C. Serum markers for allergic bronchopulmonary aspergillosis in cystic fibrosis: State of the art and further challenges. Med Mycol. 2010; 48: S77-S87.

14. Agarwal R, Aggarwal AN, Sehgal I, Dhooria S, Behera D, Chakrabarti A. Performance of serum galactomannan in patients with allergic bronchopulmonary aspergillosis. Mycoses. 2015; 58: 408-1.

15. Warren TA, Yau Y, Ratjen F, Tullis E, Waters V. Serum galactomannan in cystic fibrosis patients colonized with Aspergillus species. Med Mycol. 2012; 50: 658-660.

16. Fayemiwo S, Moore CB, Foden P, Denning DW, Richardson MD. Comparative performance of Aspergillus galactomannan ELISA and PCR in sputum from 
patients with ABPA and CPA. J Microbiol Methods. 2017; 140: 32-39.

17. Agarwal R. Allergic Bronchopulmonary Aspergillosis. Chest. 2009; 135: 805826

18. De Oliveira E, Giavina-Bianchi P, Fonseca LA, Kalil J. Allergic bronchopulmonary aspergillosis' diagnosis remains a challenge. Resp Med. 2007; 101: 2352-2357.

19. Almeida MB, Bussamra MH, Rodrigues JC. ABPA diagnosis in cystic fibrosis patients: the clinical utility of IgE specific to recombinant Aspergillus fumigatus allergens. J Paediatr. 2006; 82: 215-220.

20. Katelari A, Tzanoudaki M, Noni M, Kanariou $M$ et al. The role of basophil activation test in allergic bronchopulmonary aspergillosis and Aspergillus fumigatus sensitization in cystic fibrosis patients. J Cyst Fibros. 2016; 15: 587596.

21. Stevens DA, Schwartz HJ, Lee JY et al. A randomized trial of itraconazole in allergic bronchopulmonary aspergillosis. N Engl J Med. 2000; 342:756-762.

22. Elphick HE, Southern KW. Antifungal therapies for allergic bronchopulmonary aspergillosis in people with cystic fibrosis. Cochrane Database Syst. Rev. 2012; 6:CD002204.

23. Howard SJ, Pasqualotto AC, Denning DW. Azole resistance in allergic bronchopulmonary aspergillosis and Aspergillus bronchitis. Clin Microbiol Infect. 2010; 16: 683-688.

24. Howard SJ, Cerar D, Anderson MJ, et al. Frequency and evolution of Azole resistance in Aspergillus fumigatus associated with treatment failure. Emerg Infect Dis. 2009; 15: 1068-1076. 
25. Burgel PR, Baixench MT, Amsellem M et al. High prevalence of azole-resistant Aspergillus fumigatus in adults with cystic fibrosis exposed to itraconazole. Antimicrob Agents Chemother. 2012; 56: 869-874.

26. The UK Cystic Fibrosis Trust Microbiology Laboratory Standards Working Group. Laboratory Standards for Processing Microbiological Samples from People with Cystic Fibrosis. First edition. September 2010. https://www.cysticfibrosis.org.uk/ /media/documents/the-work-wedo/care/consensus-docs-with-new-address/laboratory-standards.ashx?la=en.

27. Normansell R, Walker S, Milam SJ, Walters EH, Nair P. Omalizumab for asthma in adults and children. Cochrane Database Syst Rev. 2014; 1: CD003559.

28. Tanou K, Zintzaras E, Kaditis AG. Omalizumab therapy for allergic bronchopulmonary aspergillosis in children with cystic fibrosis: a synthesis of published evidence. Paediatr Pulmonol. 2014; 49: 503-507.

29. Baxter CG, Moore CB, Jones AM, Webb AK, Denning DW. IgE-mediated immune responses and airway detection of Aspergillus and Candida in adult cystic fibrosis. Chest. 2013; 143: 1351-1357.

30. Shoseyov D, Brownless KG, Conway SP, Kerem E. Aspergillus bronchitis in cystic fibrosis. Chest. 2006; 130: 222-226.

31. Nagano Y, Cherie Millar B, Goldsmith CE, Elborn JS, Rendall J, Moore JE. Emergence of Scedosporium apiospermum in patients with cystic fibrosis. Arch Dis Child. 2007; 92: 607.

32. Symoens F, Knoop C, Schrooyen M, et al. Disseminated Scedosporium apiospermum infection in a cystic fibrosis patient after double-lung 
transplantation. J Heart Lung Transplant. 2006; 25: 603-607.

33. Cimon B, Carrère J, Vinatier JF, Chazalette JP, Chabasse D, Bouchara JP. Clinical significance of Scedosporium apiospermum in patients with cystic fibrosis. Eur J Clin Microbiol Infect Dis. 2000; 19: 53-56.

34. Padoan R, Poli P, Colombrita D, Borghi E, Timpano S, Berlucchi M. Acute Scedosporium apiospermum endobronchial infection in cystic fibrosis. Pediatr Infect Dis J. 2016; 35: 701-702.

35. Parize P, Billaud S, Bienvenu AL et al. Impact of Scedosporium apiospermum complex seroprevalence in patients with cystic fibrosis. J Cyst Fibros. 2014; 13: 667-673. 
Figure 1: This figure shows the differences between adult physicians $(n=32)$ and paediatricians $(n=25)$ in their treatment choices for ABPA diagnosed for the first time. Treatment choices consisted of prednisolone alone $(47 \%$ adult physicians vs $20 \%$ paediatricians used this regimen, $\mathrm{p}<0.0001$ ), prednisolone (pred) and intraconazole capsules ( $41 \%$ adult physicians vs $24 \%$ paediatricians) or itraconazole liquid ( $12.5 \%$ adult physicians vs $60 \%$ paediatricians), pred and voriconazole (was chosen only by $8 \%$ of paediatricians).

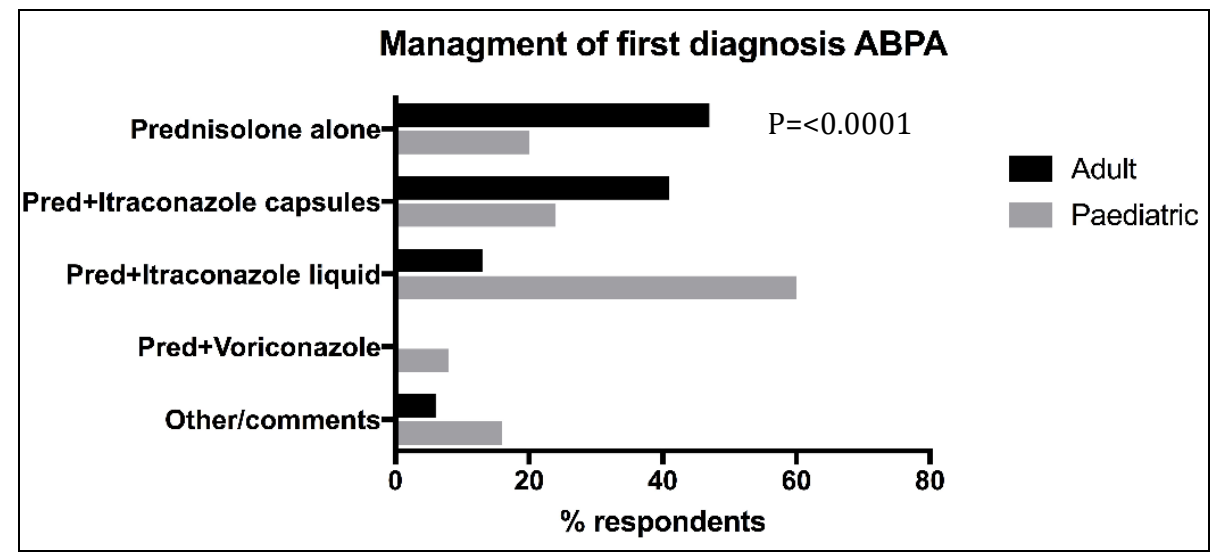


Figure 2: This figure shows the differences between adult physicians $(n=32)$ and paediatricians $(n=25)$ in their treatment choices for a first relapse of ABPA.

Treatment choices consisted of prednisolone alone $(12.5 \%$ adult physicians vs $4 \%$ paediatricians used this regimen, $\mathrm{p}<0.0001$ ), prednisolone (pred) and intraconazole capsules ( $34 \%$ adult physicians vs $12 \%$ paediatricians) or itraconazole liquid (28\% adult physicians vs $40 \%$ paediatricians), pred and voriconazole ( $16 \%$ adult physicians vs $8 \%$ paediatricians).

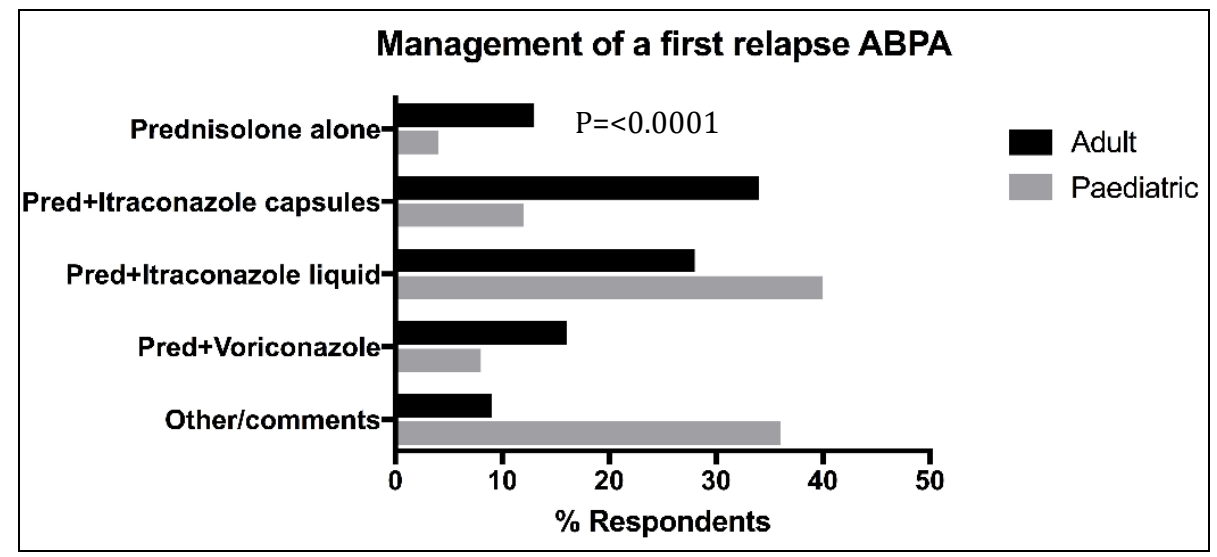


Figure 3: This figure shows how physicians reduce treatment for ABPA when clinically stable on steroid and azole therapy.

How to you reduce treatment when stable?

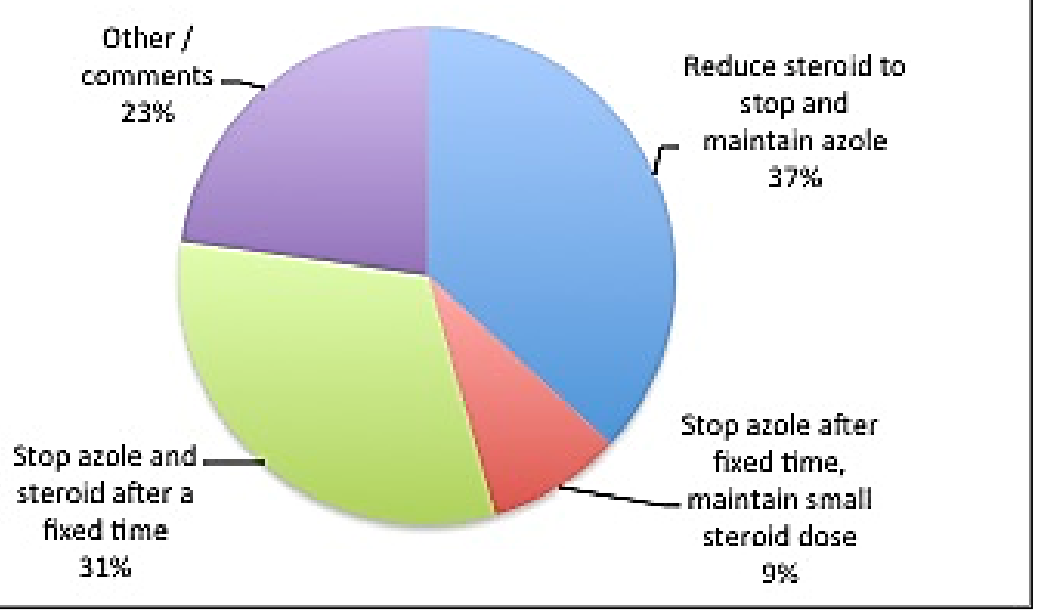

